\title{
The N-Heterocyclic Carbene Catalyzed Nucleophilic Substitution Reaction for Construction of Benzopyrones and Benzofuranones
}

Jinmei He, Jiyue Zheng, Jian Liu, Xuegong She* and Xinfu Pan*

Department of Chemistry, State Key Laboratory of Applied Organic Chemistry, Lanzhou University, Lanzhou, 730000, P. R. China

State Key Laboratory for Oxo Synthesis and Selective Oxidation, Lanzhou Institute of Chemical Physics, Chinese Academy of Sciences, Lanzhou 730000, P. R. China

shexg@,1zu.edu.cn

\section{Supporting Information}

\section{List of contents}

General Information S 1

General Procedure $\mathrm{S} 2$

Experimental Data S3

${ }^{1} \mathrm{H}$ and ${ }^{13} \mathrm{C}$ NMR spectra of products S7

\section{General Information}

The ${ }^{1} \mathrm{H}$ and ${ }^{13} \mathrm{C}$ NMR data were recorded on a Mercury Plus-300 or a Bruker-400 $\mathrm{MHz}$ spectrometer. The chemical shifts $(\delta)$ are reported in ppm and coupling constants $(J)$ in Hz. IR spectra were recorded on a Nicolet 670 FTIR spectrophotometer and reported in wavenumbers $\left(\mathrm{cm}^{-1}\right)$. Mass spectral (MS) data were obtained on a V.G.ZAB-HS mass spectrometer. High-resolution mass spectra (HRMS) were obtained on a Bruker Daltonics APEX II 47e mass spectrometer. Column chromatography was generally performed on silica gel (200-300 mesh) and TLC inspections were on silica gel GF254 plates.

Xylene was purchased at analyticaly pure and stored over molecular sieves, if not noted especially below, other solvents were purified and dried by standard methods 
prior to use. DBU was purchased from commercial suppliers and used without further treatment. Thiazolium salts and imidazolium salt were synthesized according to the literature procedure.

\section{General Procedure}

\section{Preparation of pre-substrates:}

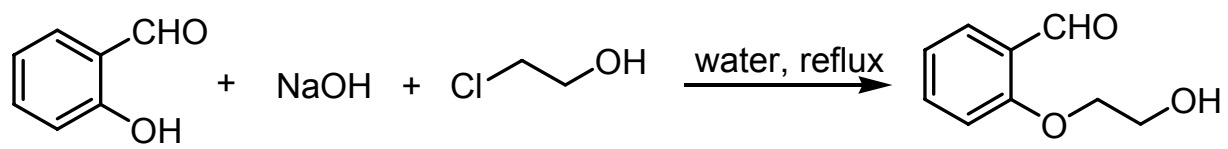

Salicylaldehyde (1.22 g, $10 \mathrm{mmol})$ was added dropwise over $15 \mathrm{~min}$ to a vigorously stirred solution of sodium hydroxide $(0.4 \mathrm{~g}, 10 \mathrm{mmol})$ in water $(10 \mathrm{ml})$, and then 2-chloroethanol (0.81 g, $10 \mathrm{mmol})$ was added dropwise and the resulting solution was heated at $98{ }^{\circ} \mathrm{C}$ for $16 \mathrm{~h}$. The solution was cooled and maintained at about $10{ }^{\circ} \mathrm{C}$ while sodium hydroxide was added until the solution was strongly alkaline $(\mathrm{PH}=10)$. The reaction mixture was extracted with dichloromethane $(4 * 20 \mathrm{ml})$. The combined organic layers were dried $\left(\mathrm{MgSO}_{4}\right)$ and filtered, concentrated in vacuo, the residue was chromatographed using 5:1 petroleum ether/ EtOAc to afford $1.262 \mathrm{~g} \mathrm{(76 \% )} \mathrm{of}$ corresponding 2-(2-hydroxyethoxy)benzaldehyde as a yellow oil.

Other precursors of substrates were generated in the same way as above from salicylaldehyde counterpart and corresponding $\alpha$-halogenated alcohol.

\section{Synthesis of benzopyrone product (5):}

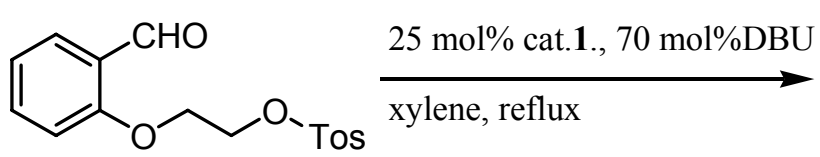

4<smiles>O=C1CCOc2ccccc21</smiles>

5

A round-bottomed flask was charged with DBU (53 $\mathrm{mg}, 0.35 \mathrm{mmol})$ in $25 \mathrm{ml}$ xylene, and thiazolium salt 1 (36 $\mathrm{mg}, 0.125 \mathrm{mmol}$ ) was added, the resulting mixture was stirred intensely at $50{ }^{\circ} \mathrm{C}$ for $15 \mathrm{~min}$, and then substrate 4 (160 mg, $0.5 \mathrm{mmol}$ ) was added. The reaction mixture was stirred $4 \mathrm{~h}$ at reflux temperature. The solvent was removed under ordinary pressure, the residue was chromatographed using 2:1 dichloromethane/petroleum ether to afford $53 \mathrm{mg}(72 \%)$ of chromanone 5 as a yellow 
oil.

Synthesis of other benzopyrones and benzofuranones were following this general procedure as above.

\section{Experimental Data}<smiles>O=C1CCOc2ccccc21</smiles>

chromanone (5):

${ }^{1} \mathrm{H}$ NMR $\left(300 \mathrm{MHz}, \mathrm{CDCl}_{3}\right) \delta 7.90(\mathrm{dd}, J=8.1,1.5 \mathrm{~Hz}, 1 \mathrm{H}), 7.48-7.45(\mathrm{~m}, 1 \mathrm{H})$, 7.05-6.96 (m, 2H), $4.54(\mathrm{t}, J=6.5 \mathrm{~Hz}, 2 \mathrm{H}), 2.82(\mathrm{t}, J=6.5 \mathrm{~Hz}, 2 \mathrm{H}) ;{ }^{13} \mathrm{C}$ NMR $(75$ $\left.\mathrm{MHz}, \mathrm{CDCl}_{3}\right) \delta 191.8,161.8,135.9,127.1,121.3,117.8,66.9,37.7$; IR (KBr) 1688, 1647, 1602, 1475, 1457, $1036 \mathrm{~cm}^{-1}$; Ms m/z (\%) $148\left(\mathrm{M}^{+}, 53\right), 120$ (100), 92 (60), 64 (14), $63(16)$.<smiles>COc1ccc2c(c1)C(=O)CCO2</smiles>

2, 3-dihydro-6-methoxychromen-4-one (8):

The reaction mixture was chromatographed using 2:1 dichloromethane/petroleum ether to afford $67 \mathrm{mg}(75 \%)$ of the indicated compound as a yellow oil; ${ }^{1} \mathrm{H}$ NMR (300 $\left.\mathrm{MHz}, \mathrm{CDCl}_{3}\right) \delta 7.31(\mathrm{~d}, J=3.3 \mathrm{~Hz}, 1 \mathrm{H}), 7.08(\mathrm{dd}, J=9.0,3.3 \mathrm{~Hz}, 1 \mathrm{H}), 6.91(\mathrm{~d}, J=$ $9.0 \mathrm{~Hz}, 1 \mathrm{H}), 4.49(\mathrm{t}, J=6.3 \mathrm{~Hz}, 2 \mathrm{H}), 3.81(\mathrm{~s}, 3 \mathrm{H}), 2.79(\mathrm{t}, J=6.3 \mathrm{~Hz}, 2 \mathrm{H}) ;{ }^{13} \mathrm{C} \mathrm{NMR}$ $\left(75 \mathrm{MHz}, \mathrm{CDCl}_{3}\right) \delta 191.9,156.6,153.9,125.2,121.1,119.2,107.3,67.1,55.8,37.7$; IR (KBr) 1685, 1490, 1282, $1035 \mathrm{~cm}^{-1}$; Ms m/z (\%) $178\left(\mathrm{M}^{+}, 48\right), 150$ (100), 135 (29), $107(36), 79(38)$.<smiles>O=C1CCOc2ccc(Br)cc21</smiles>

6-bromo-2, 3-dihydrochromen-4-one (10): 
The reaction mixture was chromatographed using 3:1 dichloromethane/petroleum ether to afford $86 \mathrm{mg}$ (76\%) of the indicated compound as a yellow oil; ${ }^{1} \mathrm{H}$ NMR (400 $\left.\mathrm{MHz}, \mathrm{CDCl}_{3}\right) \delta 7.99(\mathrm{~d}, J=1.6 \mathrm{~Hz}, 1 \mathrm{H}), 7.54(\mathrm{dd}, J=8.8,1.6 \mathrm{~Hz}, 1 \mathrm{H}), 6.88(\mathrm{~d}, J=$ $8.8 \mathrm{~Hz}, 1 \mathrm{H}), 4.53(\mathrm{t}, J=6.4 \mathrm{~Hz}, 2 \mathrm{H}), 2.81(\mathrm{t}, J=6.4 \mathrm{~Hz}, 2 \mathrm{H}) ;{ }^{13} \mathrm{C}$ NMR (100 MHz, $\left.\mathrm{CDCl}_{3}\right) \delta 190.4,160.7,138.6,129.6,122.5,119.9,114.1,67.1,37.4$; IR (KBr) 1693, 1598, 1474, 1273, $1028 \mathrm{~cm}^{-1}$; Ms m/z (\%) $228\left(\mathrm{M}^{+}, 58\right), 226\left(\mathrm{M}^{+}, 58\right), 200(62), 198$ (70), 172 (51), 170 (46), 91 (18), 86 (25), 84 (44), 63 (100).<smiles>COc1ccc2c(c1)OCCC2=O</smiles>

2, 3-dihydro-7-methoxychromen-4-one (12):

The reaction mixture was chromatographed using 3:1 dichloromethane/petroleum ether to afford $43 \mathrm{mg}(48 \%)$ of the indicated compound as a yellow oil; ${ }^{1} \mathrm{H}$ NMR (300 $\left.\mathrm{MHz}, \mathrm{CDCl}_{3}\right) \delta 7.83(\mathrm{~d}, J=9.0 \mathrm{~Hz}, 1 \mathrm{H}), 6.57(\mathrm{dd}, J=8.7,2.4 \mathrm{~Hz}, 1 \mathrm{H}), 6.40(\mathrm{~d}, J=2.4$ $\mathrm{Hz}, 1 \mathrm{H}), 4.50(\mathrm{t}, J=6.6 \mathrm{~Hz}, 2 \mathrm{H}), 3.82(\mathrm{~s}, 3 \mathrm{H}), 2.74(\mathrm{t}, J=6.6 \mathrm{~Hz}, 2 \mathrm{H}) ;{ }^{13} \mathrm{C} \mathrm{NMR}(75$ $\left.\mathrm{MHz}, \mathrm{CDCl}_{3}\right) \delta 190.5,165.9,163.8,128.8,115.2,109.9,100.7,67.3,55.6,37.4$; IR (KBr) 1679, 1608, 1256, 1158, $1116 \mathrm{~cm}^{-1}$; Ms m/z (\%) $178\left(\mathrm{M}^{+}, 69\right), 150$ (100), 122 (97), 107 (64), 79 (36), 63 (26).

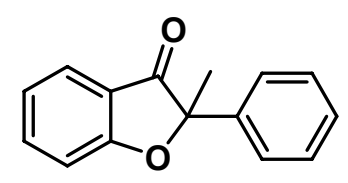

2-methyl-2-phenylbenzofuran-3(2H)-one (16):

The reaction mixture was chromatographed using 20:1 petroleum ether/EtOAc to afford $92 \mathrm{mg}(82 \%)$ of the indicated compound as a yellow oil; ${ }^{1} \mathrm{H}$ NMR $(300 \mathrm{MHz}$, $\left.\mathrm{CDCl}_{3}\right) \delta 7.69-7.59(\mathrm{~m}, 4 \mathrm{H}), 7.39-7.31(\mathrm{~m}, 3 \mathrm{H}), 7.24(\mathrm{~d}, J=8.1 \mathrm{~Hz}, 1 \mathrm{H}), 7.12-7.09$ $(\mathrm{m}, 1 \mathrm{H}), 1.85(\mathrm{~s}, 3 \mathrm{H}) ;{ }^{13} \mathrm{C} \mathrm{NMR}\left(75 \mathrm{MHz}, \mathrm{CDCl}_{3}\right) \delta 201.5,171.1,138.1,138.0,128.5$, 128.0, 125.1, 124.6, 122.0, 119.3, 113.2, 89.4, 24.2; IR (KBr) 1720, 1611, 1462, 1322 , 1254, $756 \mathrm{~cm}^{-1}$; Ms m/z (\%) 224 (M+, 100), 223 (32), 209 (16), 195 (48), 121 (69), 103 (72), 94 (74), 77 (76); HRMS (ESI) Calcd. for $\mathrm{C}_{15} \mathrm{H}_{13} \mathrm{O}_{2}[\mathrm{M}+\mathrm{H}]^{+} 225.0910$, found 


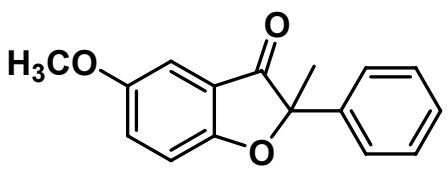

5-methoxy-2-methyl-2-phenylbenzofuran-3(2H)-one (19):

The reaction mixture was chromatographed using 20:1 petroleum ether/EtOAc to afford $102 \mathrm{mg}(80 \%)$ of the indicated compound as a yellow oil; ${ }^{1} \mathrm{H}$ NMR $(300 \mathrm{MHz}$, $\left.\mathrm{CDCl}_{3}\right) \delta 7.57(\mathrm{dd}, J=8.1,1.4 \mathrm{~Hz}, 2 \mathrm{H}), 7.36-7.27(\mathrm{~m}, 4 \mathrm{H}), 7.15(\mathrm{~d}, J=8.7 \mathrm{~Hz}, 1 \mathrm{H})$, $7.04(\mathrm{~d}, J=3.0 \mathrm{~Hz}, 1 \mathrm{H}), 3.78(\mathrm{~s}, 3 \mathrm{H}), 1.83(\mathrm{~s}, 3 \mathrm{H}) ;{ }^{13} \mathrm{C} \mathrm{NMR}\left(75 \mathrm{MHz}, \mathrm{CDCl}_{3}\right) \delta$ 201.8, 166.5, 155.0, 138.3, 128.5, 128.2, 128.0, 124.6, 119.2, 114.2, 104.6, 90.2, 55.8, 24.2; IR (KBr) 1712, 1489, 1441, 1276, 1224, 1027, $747 \mathrm{~cm}^{-1}$; Ms m/z (\%) $254\left(\mathrm{M}^{+}\right.$, 59), 253 (26), 151 (96), 124 (62), 109 (29), 103 (67), 84 (58), 77 (100), 63 (66), 51 (63); HRMS (ESI) Calcd. for $\mathrm{C}_{16} \mathrm{H}_{15} \mathrm{O}_{3}[\mathrm{M}+\mathrm{H}]^{+}$255.1021, found 255.1026.<smiles>CC1(c2ccccc2)Oc2ccc(Br)cc2C1=O</smiles>

5-bromo-2-methyl-2-phenylbenzofuran-3(2H)-one (21):

The reaction mixture was chromatographed using 20:1 petroleum ether/EtOAc to afford $130 \mathrm{mg}(86 \%)$ of the indicated compound as a yellow oil; ${ }^{1} \mathrm{H}$ NMR $(300 \mathrm{MHz}$, $\left.\mathrm{CDCl}_{3}\right) \delta 7.78-7.71(\mathrm{~m}, 2 \mathrm{H}), 7.56-7.53(\mathrm{~m}, 2 \mathrm{H}), 7.36-7.33(\mathrm{~m}, 3 \mathrm{H}), 7.14(\mathrm{~d}, J=9.0$ $\mathrm{Hz}, 1 \mathrm{H}), 1.83(\mathrm{~s}, 3 \mathrm{H}) ;{ }^{13} \mathrm{C} \mathrm{NMR}\left(75 \mathrm{MHz}, \mathrm{CDCl}_{3}\right) \delta 200 . .1,169.9,140.7,137.6$, 128.6, 128.3, 127.6, 124.6, 121.2, 115.1, 114.5, 90.6, 24.3; IR (KBr) 1727, 1605, 1462, 1273, 1160, 820, $699 \mathrm{~cm}^{-1}$; Ms m/z (\%) $304\left(\mathrm{M}^{+}, 11\right), 302\left(\mathrm{M}^{+}, 11\right), 223(23), 201$ (16), 199 (16), 174 (17), 172 (23), 103 (100), 77 (84), 63 (53); HRMS (ESI) Calcd. for $\mathrm{C}_{15} \mathrm{H}_{12} \mathrm{BrO}_{2}[\mathrm{M}+\mathrm{H}]^{+}$303.0020, found 303.0016.<smiles>COc1ccc2c(c1)OC(C)(c1ccc(Cl)cc1)C2=O</smiles> 
2-(4-chlorophenyl)-6-methoxy-2-methylbenzofuran-3(2H)-one (23):

The reaction mixture was chromatographed using 20:1 petroleum ether/EtOAc to afford $103 \mathrm{mg}$ (71\%) of the indicated compound as a yellow oil; ${ }^{1} \mathrm{H}$ NMR (400 MHz, $\left.\mathrm{CDCl}_{3}\right) \delta 7.54(\mathrm{dd}, J=8.6,7.8 \mathrm{~Hz}, 1 \mathrm{H}), 7.50(\mathrm{~d}, J=7.4 \mathrm{~Hz}, 2 \mathrm{H}), 7.32(\mathrm{dd}, J=8.4$, $1.2 \mathrm{~Hz}, 2 \mathrm{H}), 6.68-6.63(\mathrm{~m}, 2 \mathrm{H}), 3.91(\mathrm{~s}, 3 \mathrm{H}), 1.79$ (s, 3H); ${ }^{13} \mathrm{C}$ NMR (100 MHz, $\left.\mathrm{CDCl}_{3}\right) \delta 198.8,173.6,168.7,137.2,134.1,128.6,126.3,126.2,112.2,112.0,96.2$, 89.9, 55.9, 24.5; IR (KBr) 1709, 1613, 1493, 1443, 1287, 1202, $831 \mathrm{~cm}^{-1}$; Ms m/z (\%) $290\left(\mathrm{M}^{+}, 7\right), 288\left(\mathrm{M}^{+}, 21\right), 151$ (100), 134 (46), 124 (31), 106 (49), 75 (34), 63 (88); HRMS (ESI) Calcd. for $\mathrm{C}_{16} \mathrm{H}_{14} \mathrm{ClO}_{3}[\mathrm{M}+\mathrm{H}]^{+}$289.0631, found 289.0625.

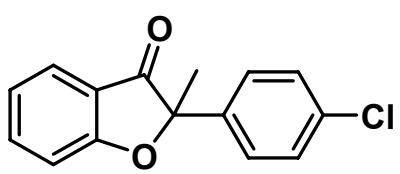

2-(4-chlorophenyl)-2-methylbenzofuran-3(2H)-one (25):

The reaction mixture was chromatographed using 20:1 petroleum ether/EtOAc to afford $100 \mathrm{mg}(77 \%)$ of the indicated compound as a yellow oil; ${ }^{1} \mathrm{H}$ NMR $(300 \mathrm{MHz}$, $\left.\mathrm{CDCl}_{3}\right) \delta 7.69-7.64(\mathrm{~m}, 2 \mathrm{H}), 7.53(\mathrm{~d}, J=8.4 \mathrm{~Hz}, 2 \mathrm{H}), 7.32(\mathrm{~d}, J=8.4 \mathrm{~Hz}, 2 \mathrm{H}), 7.22$ $(\mathrm{d}, J=9.0 \mathrm{~Hz}, 1 \mathrm{H}), 7.10(\mathrm{t}, J=7.2 \mathrm{~Hz}, 1 \mathrm{H}), 1.81(\mathrm{~s}, 3 \mathrm{H}) ;{ }^{13} \mathrm{C} \mathrm{NMR}\left(75 \mathrm{MHz}, \mathrm{CDCl}_{3}\right)$ $\delta 201.1,171.0,138.4,136.7,134.1,128.7,126.2,125.2,122.3,119.2,113.4,88.9$, 24.4; IR 1721,1611, 1482, 1091, $757 \mathrm{~cm}^{-1}$; Ms m/z (\%) $260\left(\mathrm{M}^{+}, 11\right), 258\left(\mathrm{M}^{+}, 35\right)$, 139 (36), 137 (38), 121 (100), 94 (93), 76 (60); HRMS (ESI) Calcd. for $\mathrm{C}_{15} \mathrm{H}_{13} \mathrm{ClO}_{2}$ $[\mathrm{M}+\mathrm{H}]^{+}$259.0526, found 259.0530. 
$86 L^{\circ} 2$
$618^{\circ} 2$

$68^{\circ} 2$
$\operatorname{ta~} 8 \cdot 2$

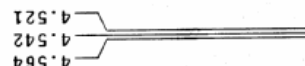

$656 \cdot 9$

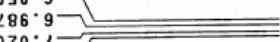

St 0.2

50.

(9)

.

LC०.

$588^{\circ} 2$

$688^{\circ} 2$

$88^{\circ}$

II 6.2

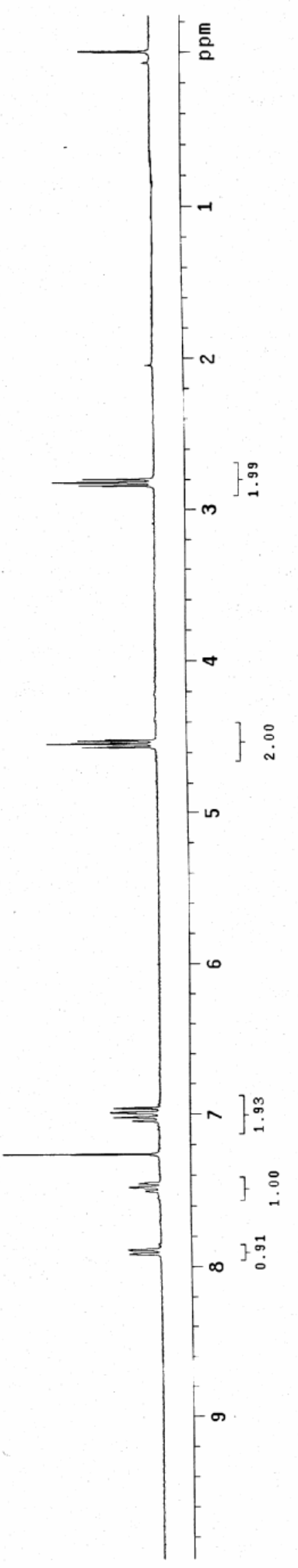


$8 \tau L \cdot \angle \varepsilon-$

056.99-

$\varepsilon<s^{\circ} 92$

$000 \cdot 22$

$\angle 28 \cdot \angle T T-$

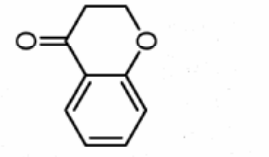

$2 \angle 0 . \angle 2 \tau-$

$006 \cdot 581-$

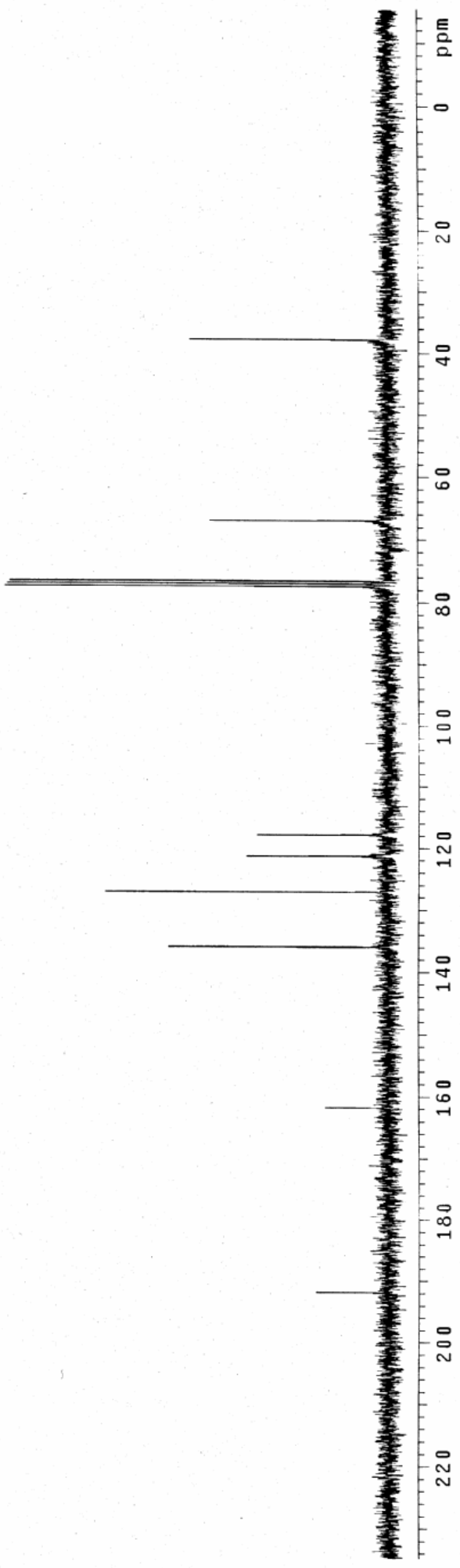

$918^{\circ}$ โ6โ 
$0<2 \cdot 2$

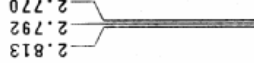

$908^{\circ} \varepsilon$

$\tau \angle b \cdot b-$

$\checkmark V^{\circ} \cdot b^{\circ}$

$068 \cdot 9-$

$026^{\circ} .9$
$b 90.2$

$S \angle 0 \cdot<$

b $60 .<$

SOI. 2

$092 \cdot 2$
$00 \varepsilon \cdot<$
$02 \varepsilon$

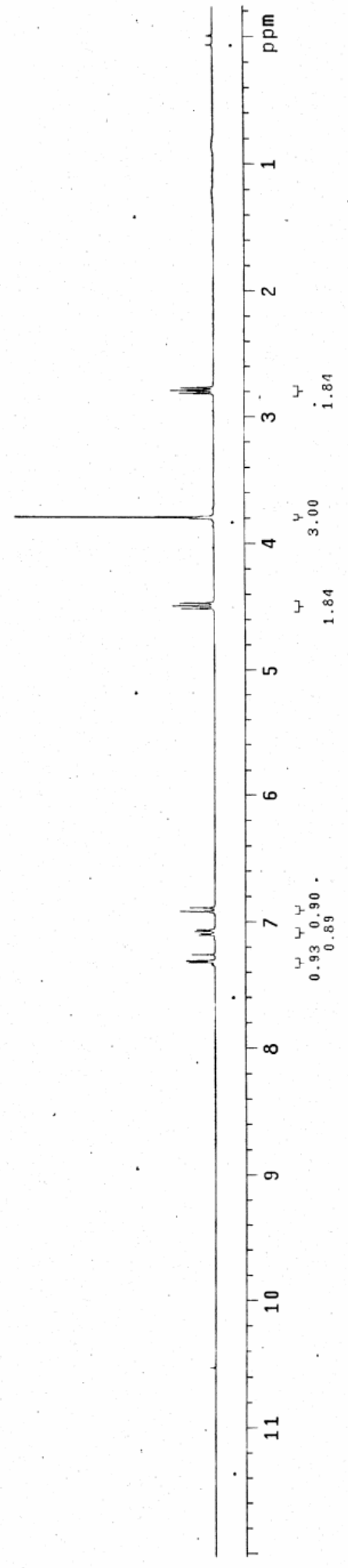



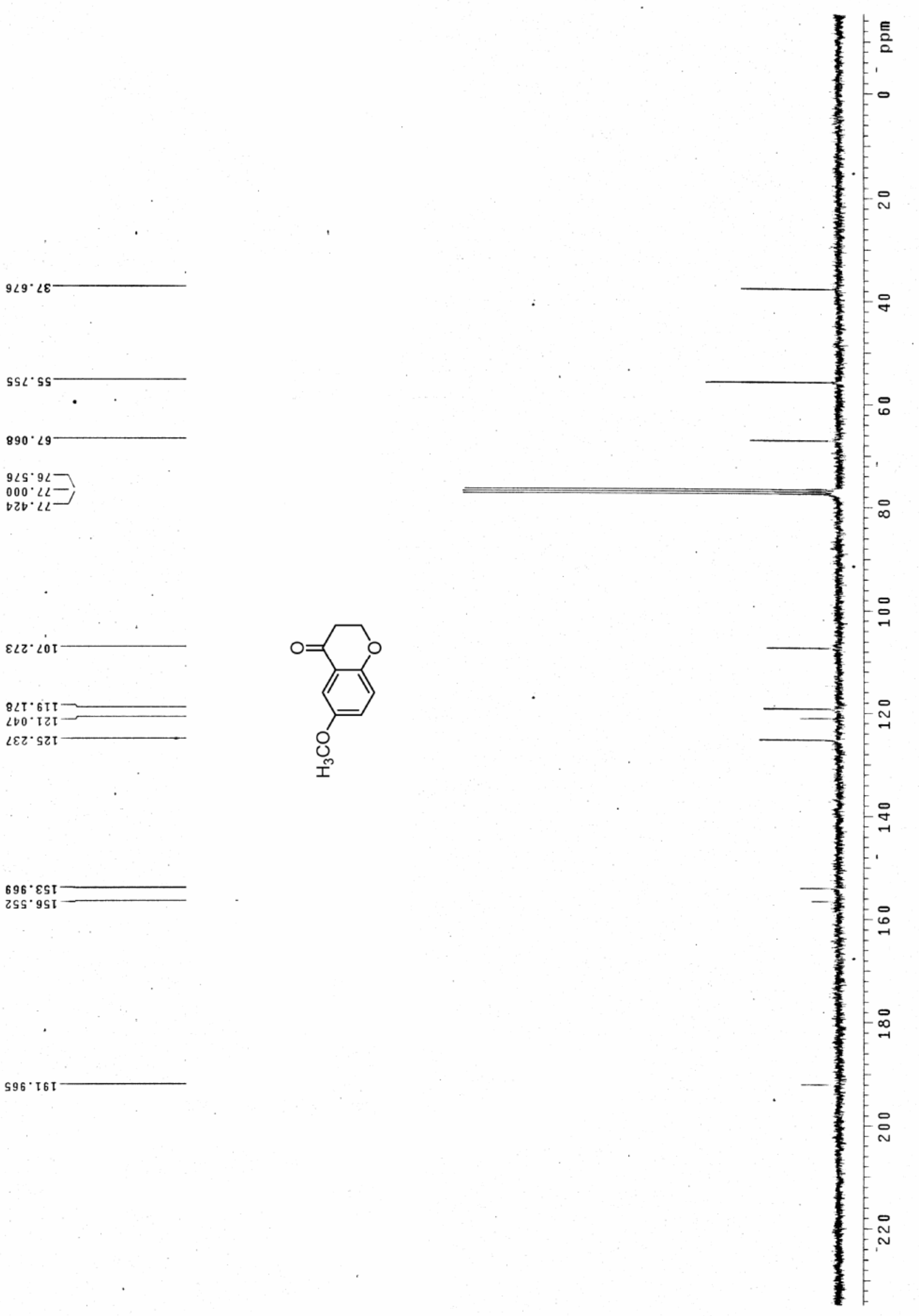

$696^{\circ} \mathrm{EST}$

$8 \angle T^{\circ} 6 \tau t$
$\angle D 0$.

LEน szI

$9 \angle S \cdot 9 L Z$
$000 \cdot 22=$
$020 \cdot 2 L$

$\varepsilon \angle \tau \cdot \angle 0 \mathrm{r}$

2SS. 9 SI

$596^{\circ} .16 \mathrm{I}$

政 


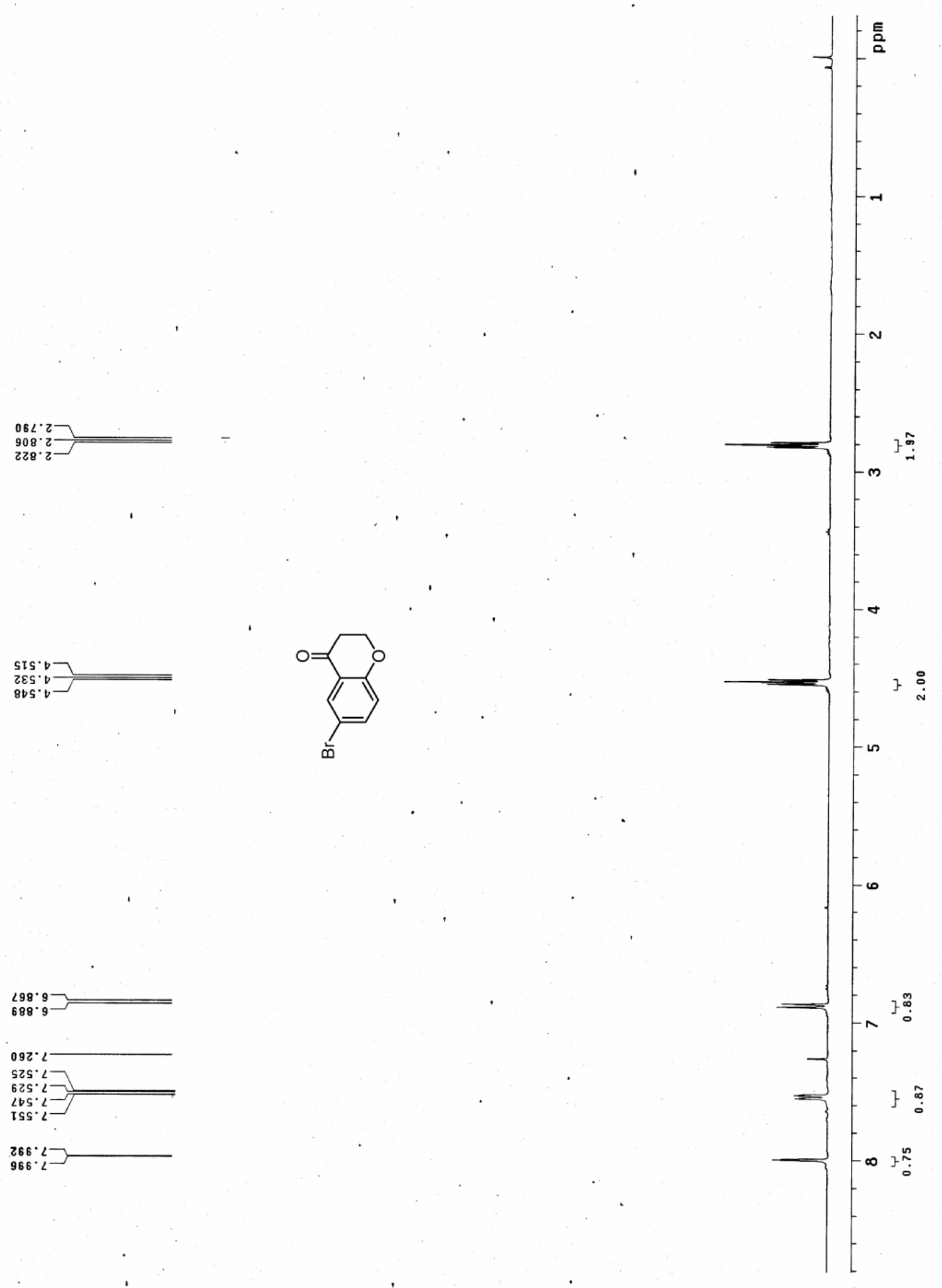




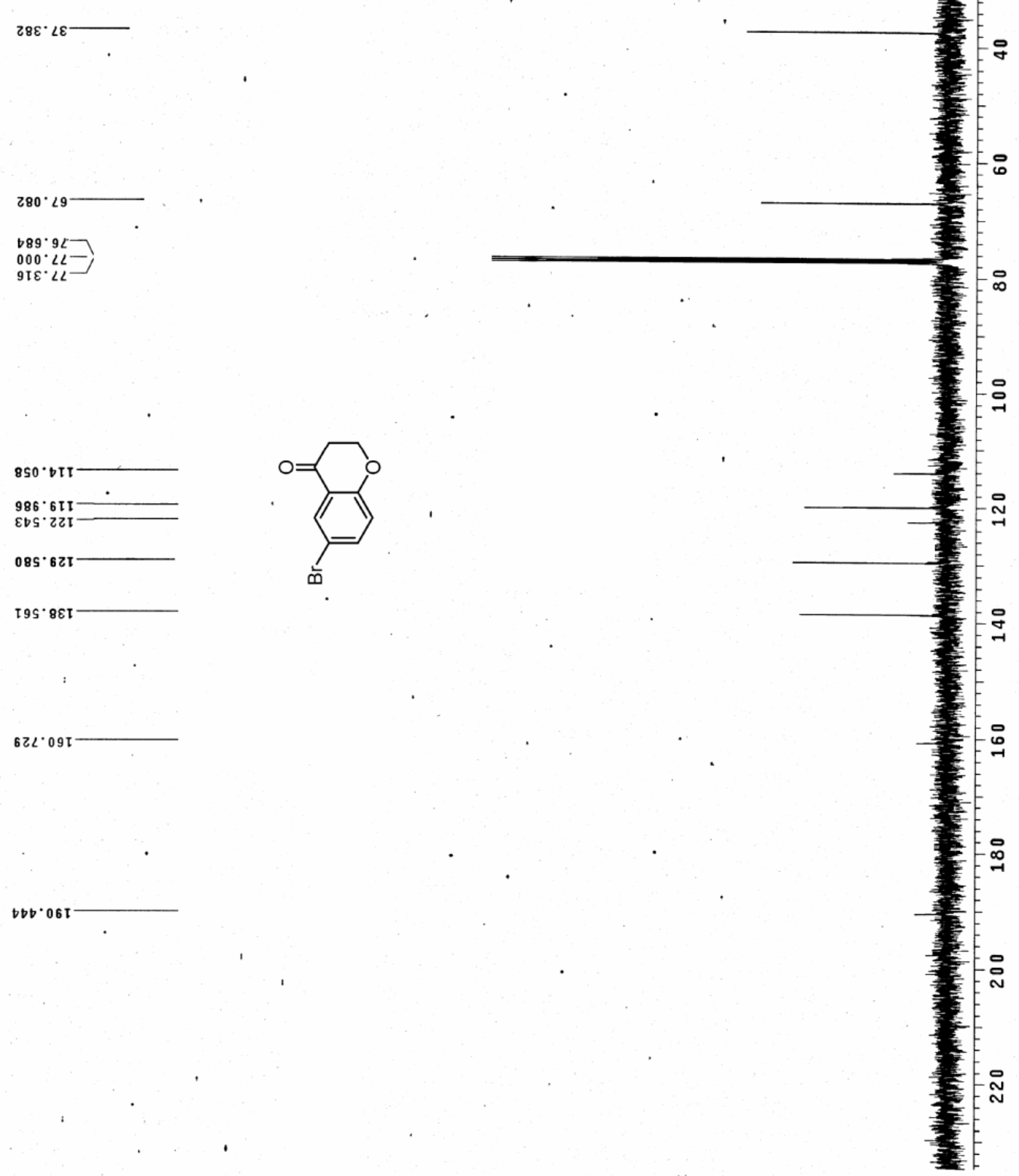


$\varepsilon z L \cdot \tau$

$\varepsilon b L^{\circ} Z$
$99 L$

$\varepsilon 乙 8^{\cdot} \varepsilon^{-}$

$980^{\circ} b^{\circ}$

$\angle 05^{\circ} \mathrm{b}-$

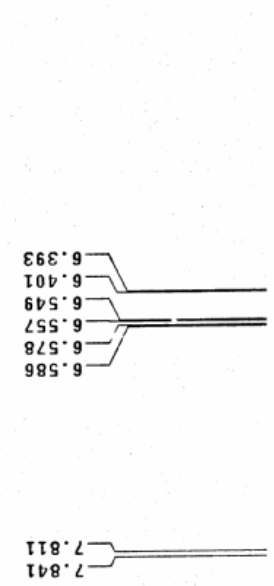

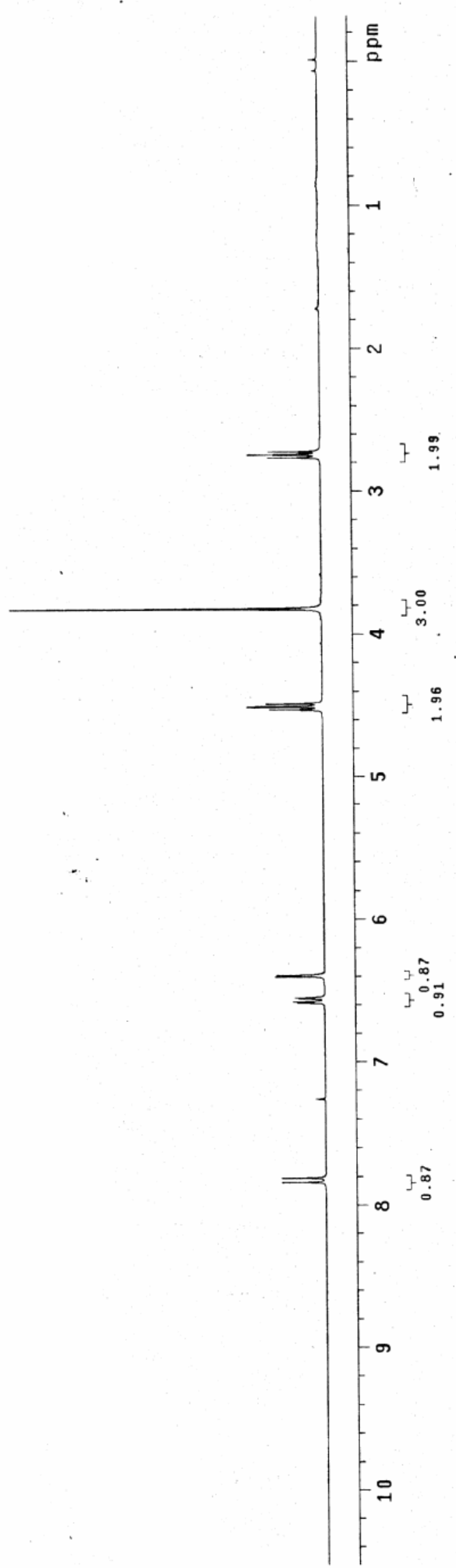




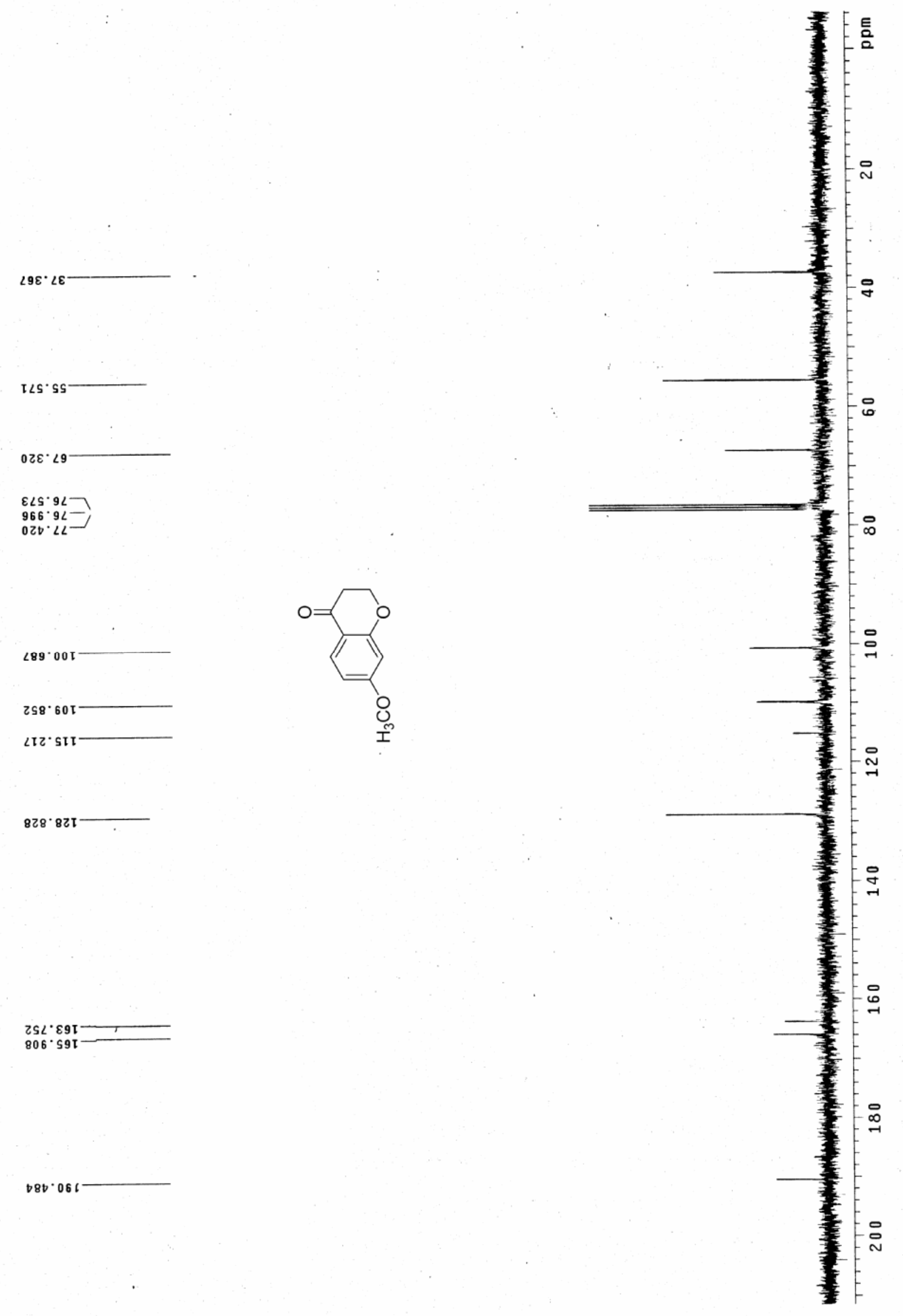

S 14 


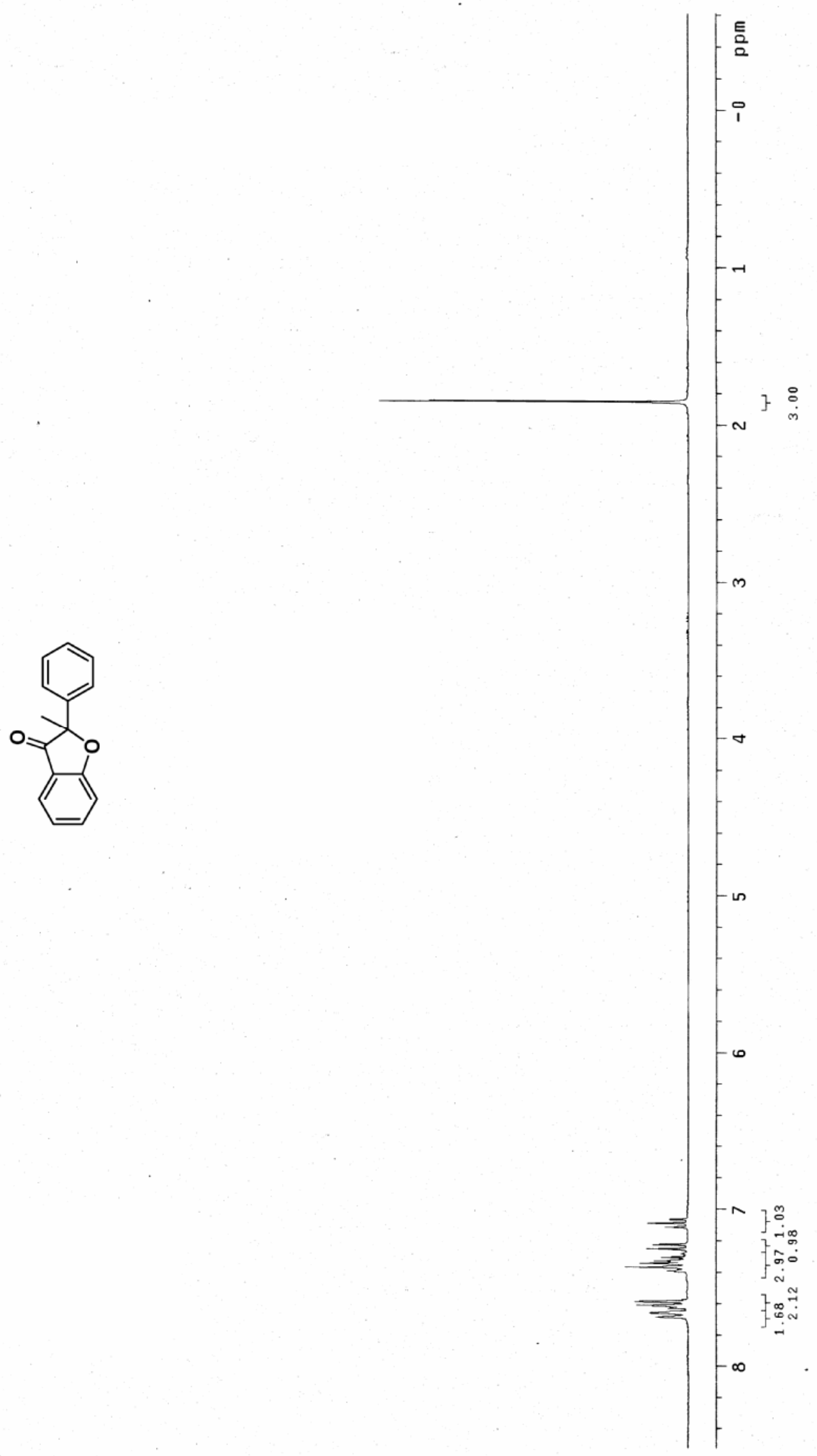

$060 \%$

$9 \mathrm{II} \cdot \mathrm{L}$
$\mathrm{SzZ} \cdot \mathrm{L}$

$252 \cdot 27$

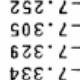

$\varepsilon \varepsilon \cdot L$

$b D \varepsilon^{\circ}<-$
$0<\varepsilon^{\circ}<-$

$\varepsilon 6 \varepsilon^{\circ}<-$

टा9.<

โ 89.2

659.2

$899^{\circ}<$
$889^{\circ} L$ 


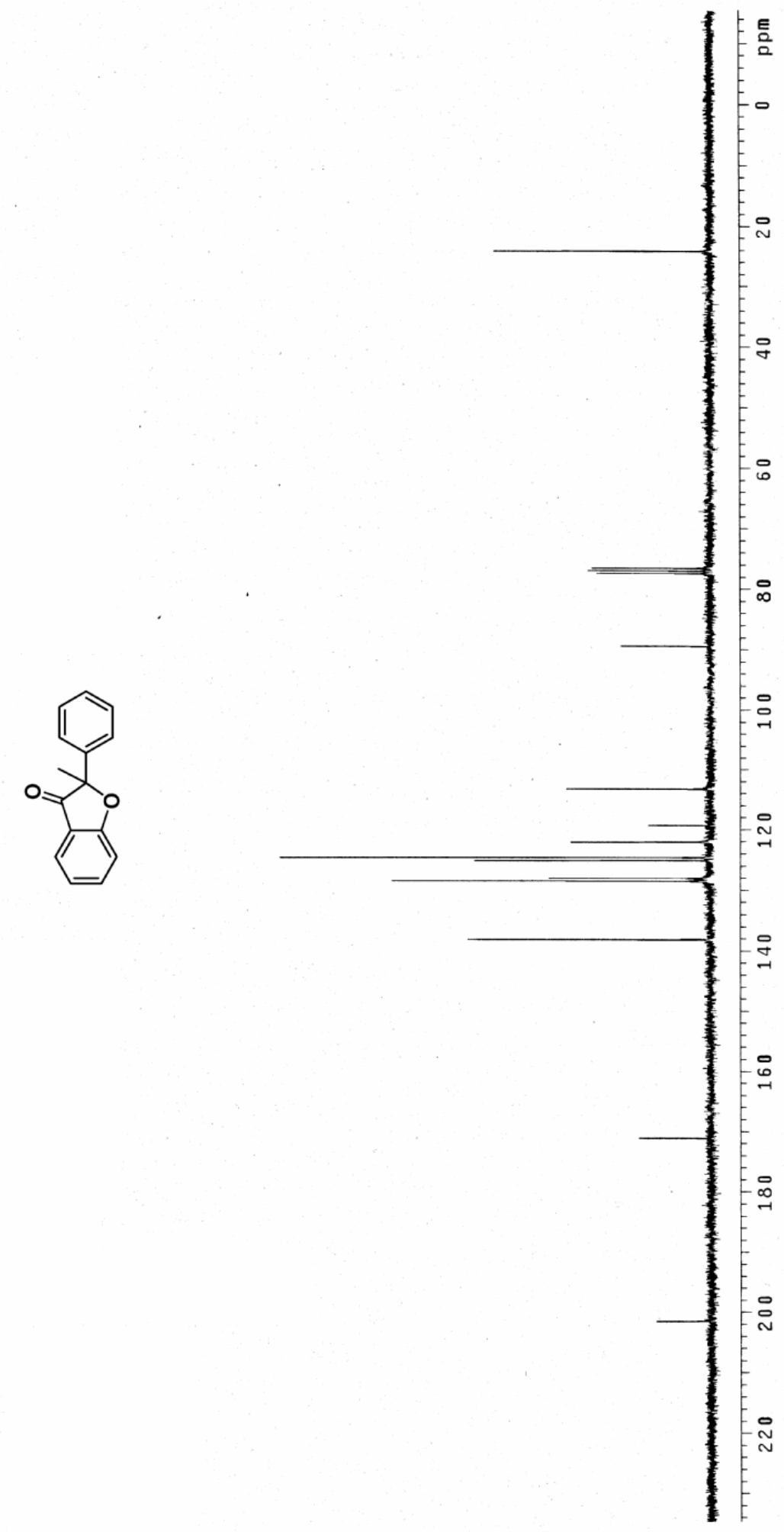


$2 \varepsilon 8^{\circ} \tau$

$28 L^{\cdot} \varepsilon$

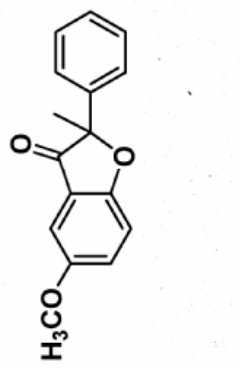

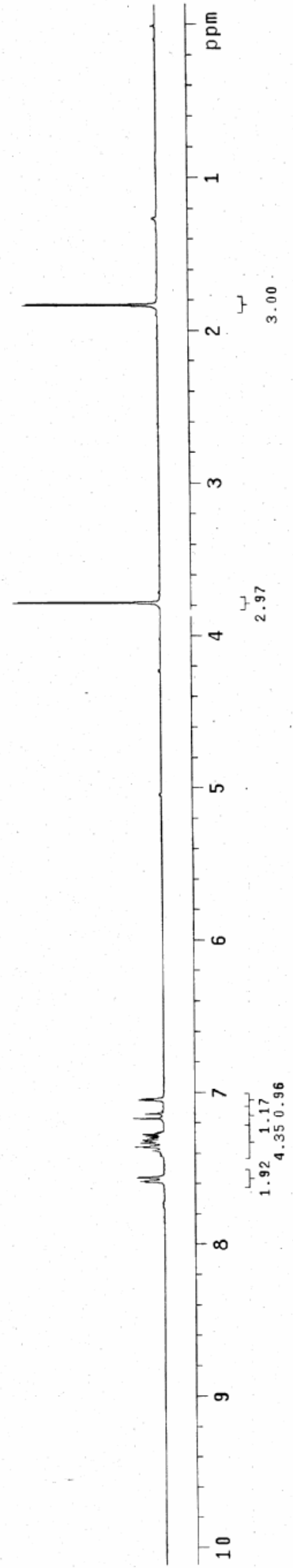




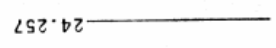

228. 55
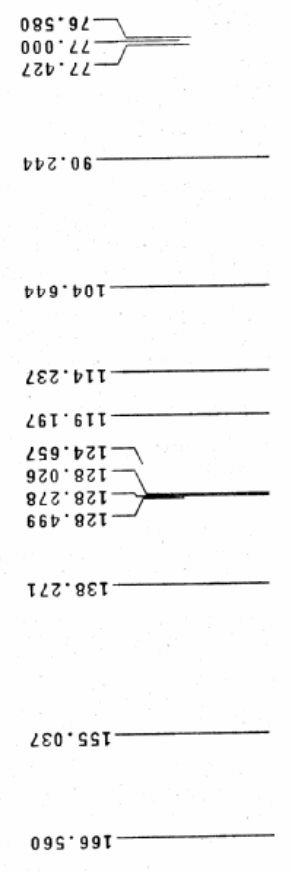

โร8. 102

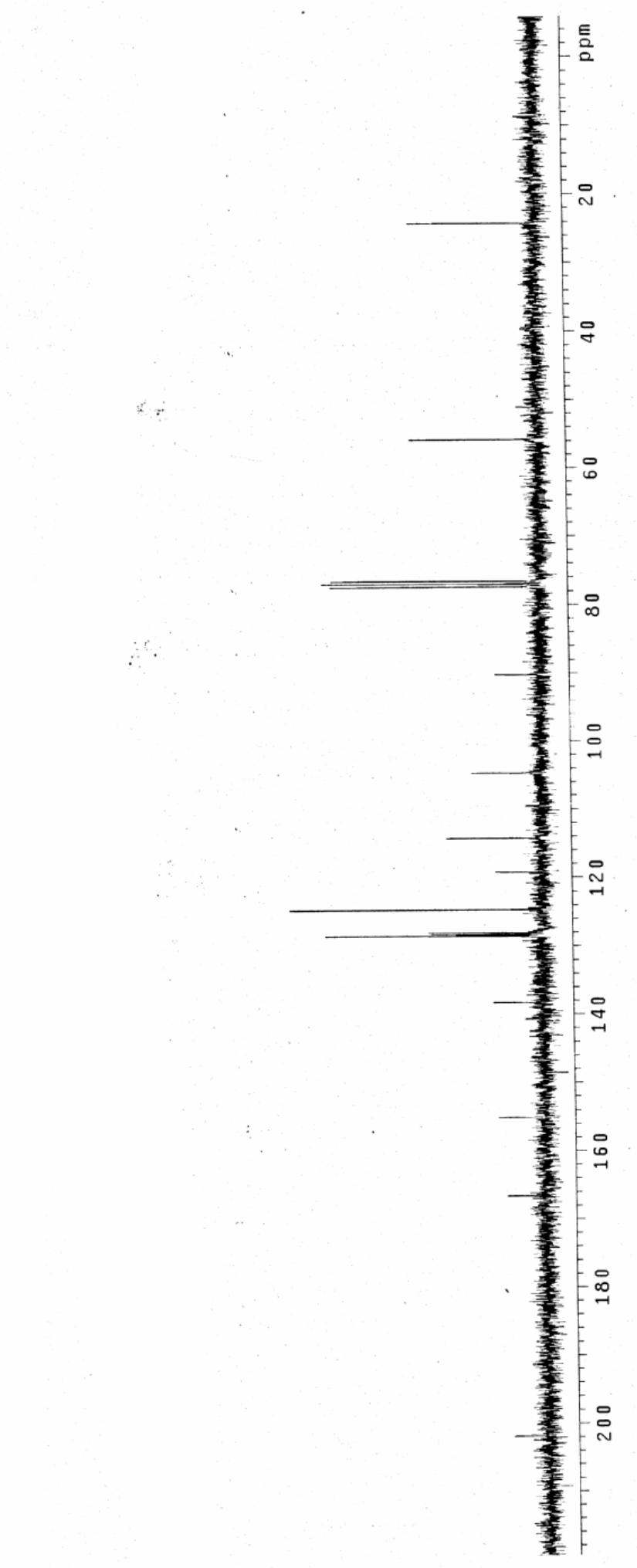

S 18 
DER'T
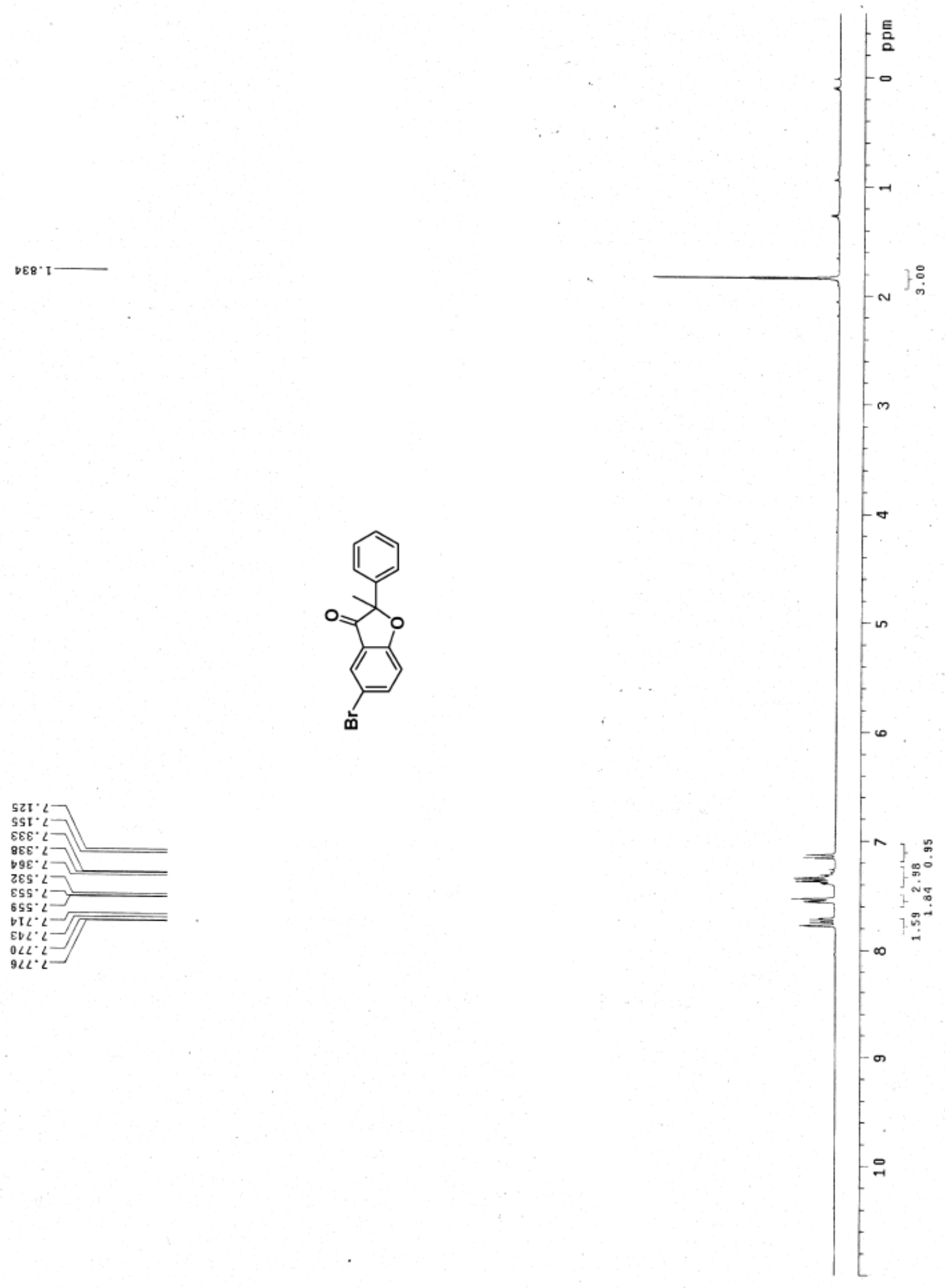

$\bar{\nu}$

S 19 


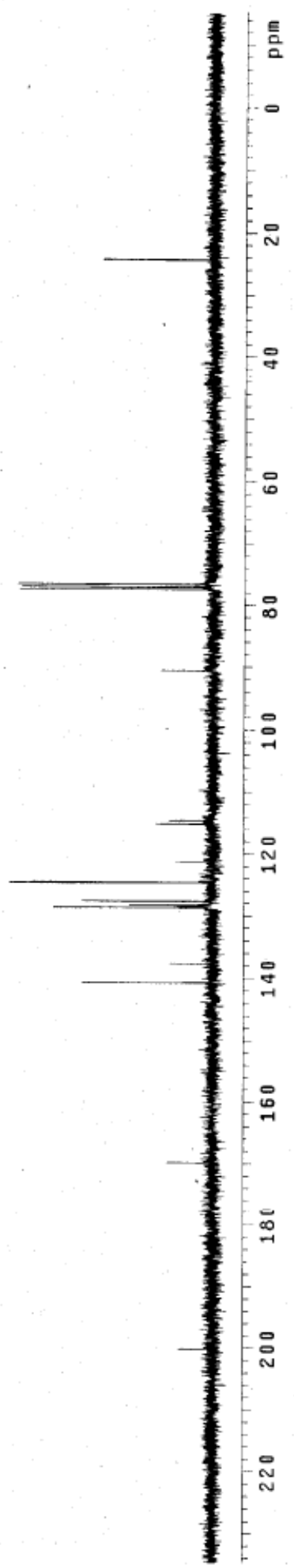

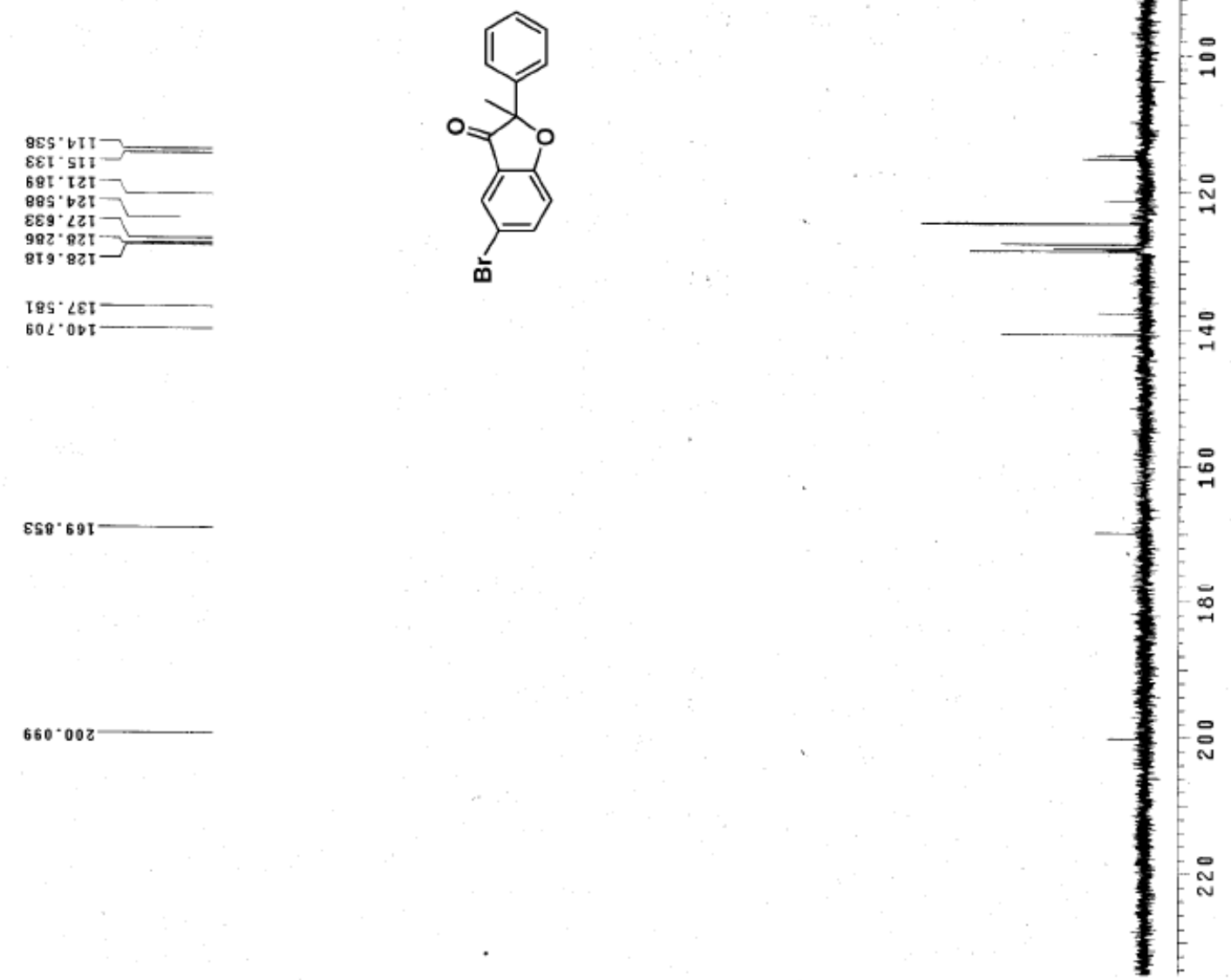

$9 \angle S^{\circ} 9 L$
$000 \cdot \angle L$

000
020

LSS.08

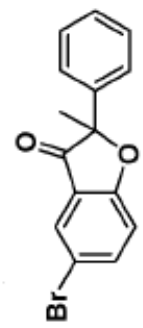

. 
$200 \cdot 0-7$

$682^{\circ} 1$

DI $6 \cdot \varepsilon$

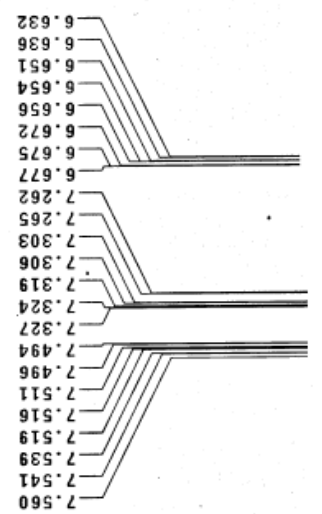

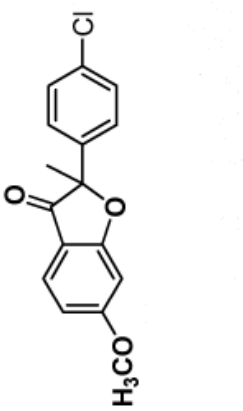

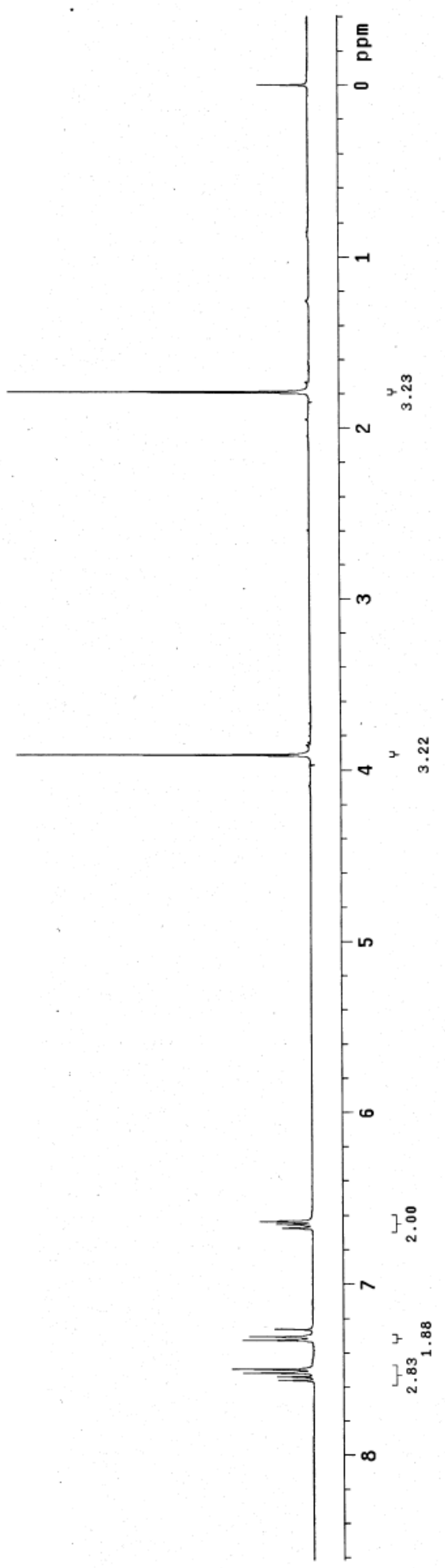


$850^{\circ}$

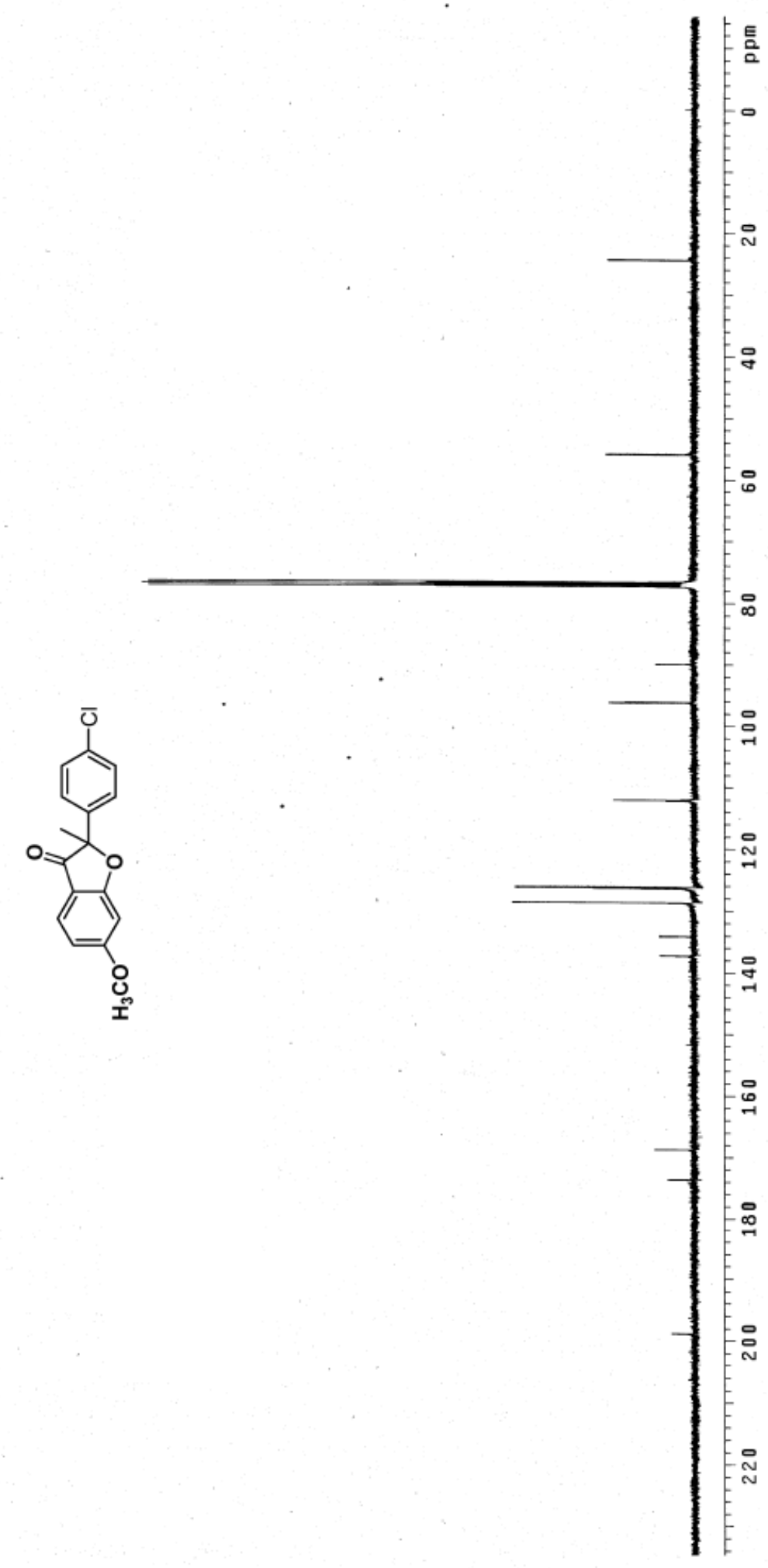

$889 \cdot 891$

$69 S^{\circ} \varepsilon \angle \tau$

9TZ. $2 \mathrm{TI}$

$\cos 98 \mathrm{ZIT}$

D80. $D E T$

$S E \tau^{\circ} \angle E \mathrm{~T}$

689.927

$686 \cdot 68$
$68 \mathrm{~T} \cdot 96$

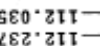

$26 L^{\circ} 86 \mathrm{I}$

$\frac{9}{4}$

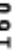

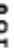

N 


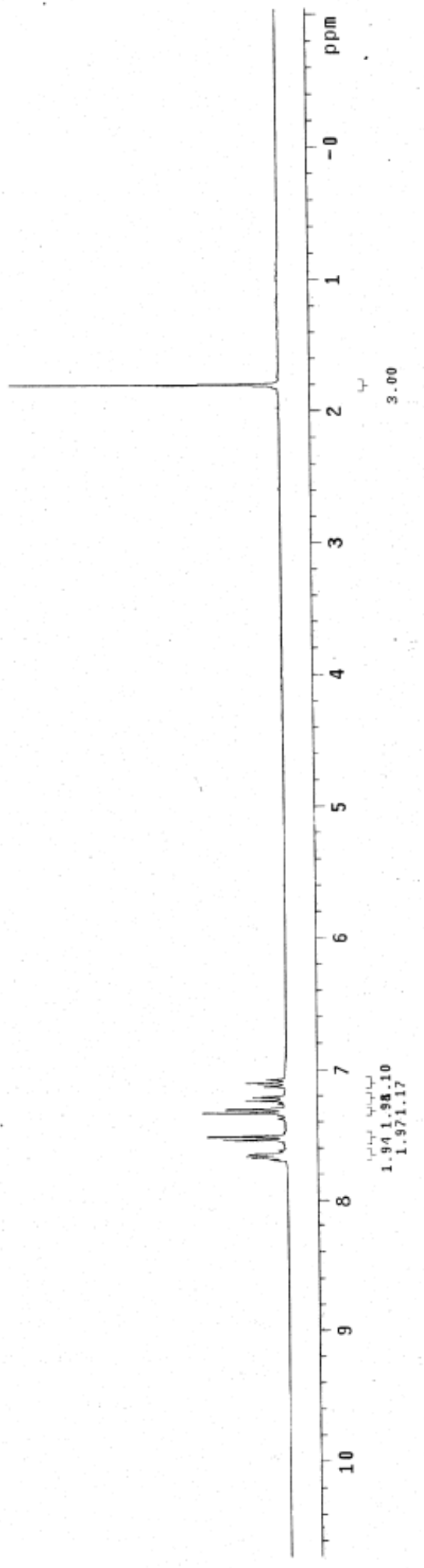




$$
\text { 列 }
$$

